\title{
Losing the stem-loop structure from metazoan mitochondrial tRNAs and co-evolution of interacting factors
}

\author{
Yoh-ichi Watanabe $^{1 *}$, Takuma Suematsu ${ }^{1+}$ and Takashi Ohtsuki ${ }^{2}$ \\ 1 Department of Biomedical Chemistry, Graduate School of Medicine, The University of Tokyo, Tokyo, Japan \\ ${ }^{2}$ Department of Biotechnology, Okayama University, Okayama, Japan
}

\section{Edited by:}

Akio Kanai, Keio University, Japan

\section{Reviewed by:}

Hyouta Himeno, Hirosaki University, Japan

Kozo Tomita, Biomedical Research Institute - National Institute of

Advanced Industrial Science and

Technology, Japan

\section{*Correspondence:}

Yoh-ichi Watanabe, Department of Biomedical Chemistry, Graduate School of Medicine, The University of Tokyo, Hongo, Bunkyo-ku, Tokyo 113-0033, Japan

e-mail:ywatanab@m.u-tokyo.ac.jp;

Takashi Ohtsuki, Department of

Biotechnology, Okayama University,

3-1-1 Tsushimanaka, Okayama

700-8530, Japan

e-mail:ohtsuk@okayama-u.ac.jp

${ }^{\dagger}$ Present address:

Takuma Suematsu, Department of Molecular and Cell Biology, Boston University, 72 East Concord Street, Boston, MA 02118, USA
Conventional tRNAs have highly conserved sequences, four-armed cloverleaf secondary structures, and L-shaped tertiary structures. However, metazoan mitochondrial tRNAs contain several exceptional structures. Almost all tRNAs ${ }^{\text {Ser }}$ for AGY/N codons lack the Darm. Furthermore, in some nematodes, no four-armed cloverleaf-type tRNAs are present: two tRNAs ${ }^{\text {Ser }}$ without the D-arm and 20 tRNAs without the T-arm are found. Previously, we showed that in nematode mitochondria, an extra elongation factor Tu (EF-Tu) has evolved to support interaction with tRNAs lacking the T-arm, which interact with C-terminal domain 3 in conventional EF-Tu. Recent mitochondrial genome analyses have suggested that in metazoan lineages other than nematodes, tRNAs without the T-arm are present. Furthermore, even more simplified tRNAs are predicted in some lineages. In this review, we discuss mitochondrial tRNAs with divergent structures, as well as protein factors, including EF-Tu, that support the function of truncated metazoan mitochondrial tRNAs.

Keywords: mitochondrial tRNA, D-arm, T-arm, tRNA nucleotidyltransferase, aminoacyl-tRNA synthetase, elongation factor Tu, ribosome

\section{INTRODUCTION}

As discussed in other articles in this special issue, conventional tRNAs are highly conserved: they have a four-armed cloverleaf secondary structure and L-shaped tertiary structure (Figures 1A,B; Jühling etal., 2009). However, some tRNAs encoded in mitochondrial DNA, particularly in metazoan (multi-cellular animal) mitochondria, have diverged from standard form tRNAs in a variety of ways. In this review, we focus on mitochondrial tRNAs (mt tRNAs) lacking either the dihydrouridine arm (D-arm) or the ribothimidine arm (T-arm; Figures 1C,D). The function of tRNA is to help decode mRNA into protein. tRNA collaborates with a variety of proteins from post-transcription to decoding in ribosomes. The unique characteristics of factors interacting with such shrunken tRNAs have been uncovered over the past several decades. In this review, these factors and their evolution will also be discussed.

\section{SHRUNKEN mt tRNAs} tRNAs LACKING THE D-arm

In the 1970s, D-arm-lacking tRNAs were at first identified as non-tRNA molecules (a putative equivalent to cytoplasmic 5S ribosomal RNA) because of their short length (Dubin and Friend, 1972; Dubin et al., 1974; Baer and Dubin, 1980). Since the identification of genes in mitochondrial DNA, these truncated tRNAs have been proposed to be functional (Arcari and Brownlee, 1980; de Bruijn et al., 1980). Virtually all metazoan mitochondria have at least one of D-arm-lacking tRNA, namely tRNA ${ }^{\text {Ser }}$ (GCU/UCU) for AGY or AGN codons (Figure 1C; Jühling et al., 2009). In addition, some animal mitochondria have additional D-arm-lacking tRNAs, such as tRNA ${ }^{\text {Ser }}$ (UGA) in chromadorean nematodes (Okimoto et al., 1992), and tRNA ${ }^{\text {Cys }}$ in some vertebrates (Seutin et al., 1994).

The secondary structures of D-arm-lacking tRNAs have been classified into several groups based on the base pairs in $\mathrm{T}$ and anticodon stems (Steinberg et al., 1994). Experimental verifications of the secondary and tertiary structures have been performed using chemical modification, limited enzymatic digestion, nuclear magnetic resonance (NMR) spectroscopy, and native gel electrophoresis (de Bruijn and Klug, 1983; Ueda et al., 1985; Hayashi et al., 1997; Frazer-Abel and Hagerman, 1999, 2004; Ohtsuki et al., 2002a). Although these results support the coaxial stacking of $T$ and acceptor stems (Frazer-Abel and Hagerman, 2008), this idea is somewhat controversial, possibly because of the structural flexibility of the D-arm-lacking tRNAs themselves (Frazer-Abel and Hagerman, 2008). The shortest possible D-arm-lacking tRNA was suggested to be $54 \mathrm{nt}$ long (Steinberg et al., 1994).

Aminoacylation and EF-Tu binding of D-arm-lacking tRNAs have been demonstrated (Ueda etal., 1985, 1992; 


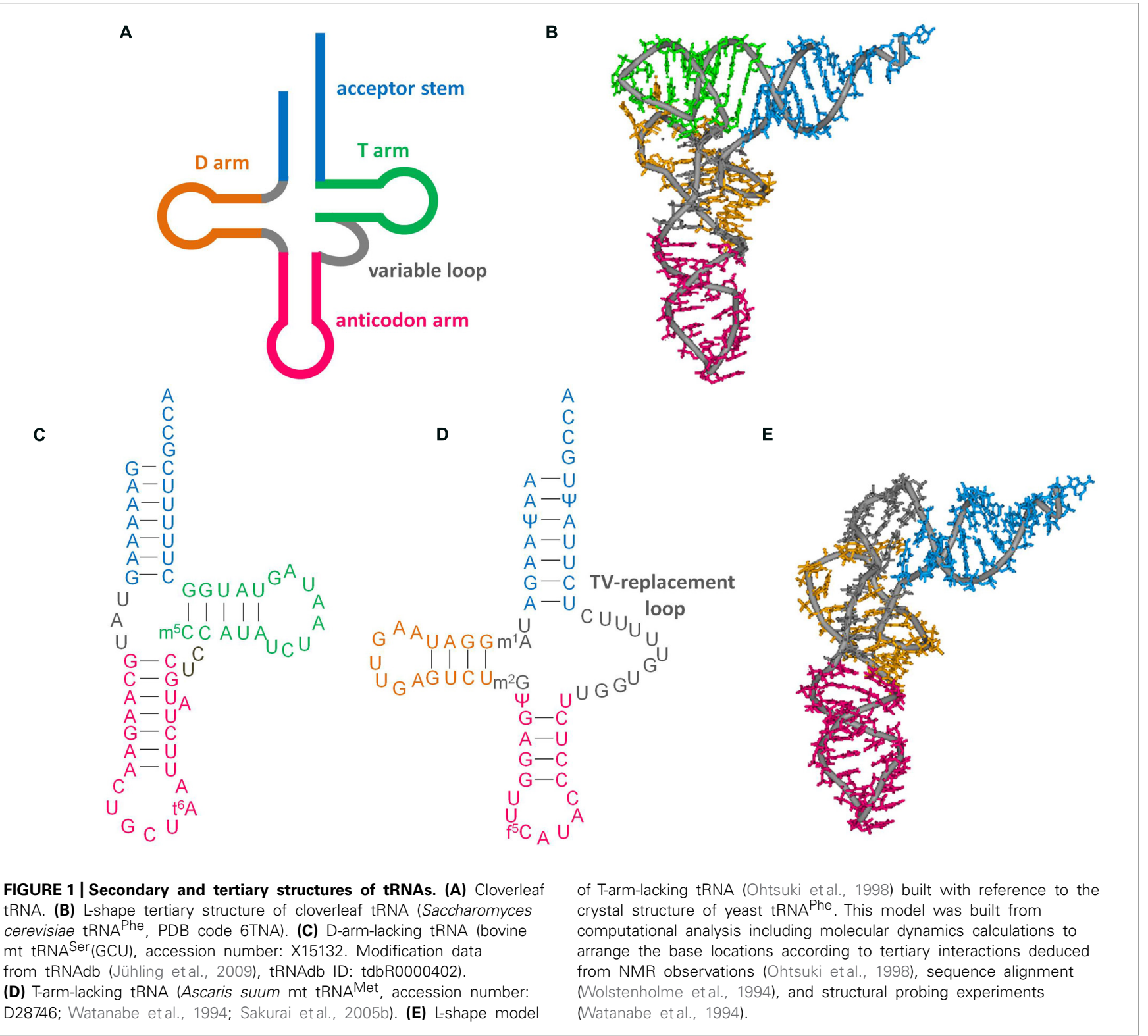

Yokogawa et al., 1989, 2000; Kumazawa et al., 1991; Watanabe et al., 1994; Hanada et al., 2001; Shimada et al., 2001; Ohtsuki etal., 2002a; Chimnaronk etal., 2005; Suematsu et al., 2005). Translation with an unmodified D-arm-lacking mammalian $\mathrm{mt}$ tRNA $^{\text {Ser }}$ (GCU) derivative with a GAA anticodon has been investigated using a cell-free system (Hanada et al., 2001). The ability to form a ternary complex with EF-Tu/GTP of the tRNA ${ }^{\text {Ser }}(\mathrm{GCU})$ derivative is similar to that of the $\mathrm{tRNA}^{\mathrm{Ser}}$ (UGA) derivative, which has a four-armed cloverleaf secondary structure (Hanada et al., 2001). However, the amount of peptides produced using $\mathrm{tRNA}^{\mathrm{Ser}}(\mathrm{GCU})$ derivative is lower than that produced using tRNA $^{\text {Ser }}$ (UGA) derivative (Hanada et al., 2001).

\section{tRNAs LACKING THE T-arm}

T-arm-lacking tRNA genes were identified in nematode mitochondria in Wolstenholme et al. (1987). Since then, T-arm-lacking
tRNA genes have also been found in mitochondrial DNA in other lineages of animals, such as Arthropoda (Masta, 2000). They have a TV replacement loop instead of a variable loop and Tarm (Figure 1D). Isolation of nematode mt T-arm-lacking tRNAs has been performed with preparative gel electrophoresis and/or solid-phase DNA affinity purification (Watanabe et al., 1994, 1997, Ohtsuki et al., 1998; Sakurai et al., 2005a,b).

Basically, intramolecular interactions in T-arm-lacking tRNAs are thought to be identical to those in conventional tRNAs, except for interactions between T- and D- arms, because of conservation around the $\mathrm{D}$-arm and the similarity of the $5^{\prime}$ region in the TV replacement loop to the variable loop region (Watanabe et al., 1994; Wolstenholme et al., 1994). As an analog of cloverleaftype tRNA, an L-shape-like structure of T-arm-lacking tRNAs has been proposed (Watanabe et al., 1994; Wolstenholme et al., 1994). Hypothesized interactions have been supported by chemical and 
enzymatic probing and NMR spectroscopy (Figure 1E; Watanabe et al., 1994; Ohtsuki et al., 1998).

The aminoacylation capacity of T-arm-lacking nematode $\mathrm{mt}$ tRNAs has been demonstrated with mt extract or recombinant enzymes (Watanabe et al., 1994; Ohtsuki et al., 1996; Chihade et al., 1998; Lovato et al., 2001; Sakurai et al., 2005b; Arita et al., 2006). Furthermore, the formation of a tertiary complex of a T-armlacking aminoacyl tRNA/EF-Tu/GTP has been detected (Ohtsuki et al., 2001; Arita et al., 2006).

In nematode mt T-arm-lacking tRNAs sequenced at the RNA level, the 1-methyladenosine at position 9 is strictly conserved (Watanabe et al., 1994, 1997; Sakurai et al., 2005a,b; see also Ohtsuki and Watanabe, 2007). This modification helps maintain the tertiary structure of the tRNA, and also aids in efficient aminoacylation and formation of the ternary complex with EF-Tu/GTP (Sakurai et al., 2005b).

\section{tRNAs POTENTIALLY LACKING BOTH D- AND T-arms}

As mentioned above, the shortest biochemically characterized tRNA is a 54-nt long mt tRNA ${ }^{\mathrm{Ser}}$ (UCU) from the nematode Ascaris suum (Watanabe et al., 1994). A computational survey of mitochondrial tRNA genes predicted the presence of tRNA genes lacking both D- and T-arms (Jühling et al., 2012a,b). More recently, after the submission of the abstract of this review, RTPCR analyses using $5^{\prime}$ - and $3^{\prime}$-RACE showed that such putative tRNA genes are indeed transcribed, and the transcripts even have a $3^{\prime} \mathrm{CCA}$ sequence in the nematode Romanomermis culicivorax (Wende et al., 2014). Note that some tRNAs are imported from the cytoplasm into the mitochondria in some animals (Rubio et al., 2008), suggesting that imported tRNAs may function in place of mitochondrial-encoded putative tRNAs lacking both D- and T-arms. Thus, functional analysis of such extremely truncated putative tRNAs is critical.

\section{FACTORS INTERACTING WITH tRNAs LACKING D- OR T-arms AMINOACYL TRNA SYNTHETASES}

Aminoacyl tRNA synthetases recognize a cognate tRNA and add an aminoacyl moiety to its $3^{\prime}$ end. The major recognition sites of the enzymes in tRNAs are the anticodon, a discriminator base at position 73, and the acceptor stem (Giegé et al., 1998). In fact, in the case of alanyl-tRNA synthetases, even the mitochondrial enzyme uses the acceptor stem as a major recognition site (Chihade et al., 1998). However, some of the enzymes also use the D-arm in tRNA [e.g., Escherichia coli isoleucyl-tRNA synthetase (Nureki etal., 1994)]. Whether the mitochondrial counterparts of such enzymes encoded in nuclear genome still use D-arms as recognition sites is an interesting issue yet to be investigated. If so, even if a tRNA lost its T-arm, the enzyme could still add the aminoacyl moiety to the shrunken tRNA.

On the other hand, seryl-tRNA synthetase (SerRS) uses recognition sites other than the anticodon and acceptor stem. Bacterial SerRS recognizes $\mathrm{T}$ - and characteristic long variable arms in bacterial tRNA ${ }^{\text {Ser }}$ (Asahara etal., 1994) using the N-terminal coiled-coil region (Biou et al., 1994). However, metazoan mt tRNA $^{\text {Ser }}$ has lost its long variable arm, and even the Darm is absent in tRNA $^{\mathrm{Ser}}$ (GCU/UCU). Thus, how mt SerRS recognizes $\mathrm{mt} \mathrm{tRNA}^{\mathrm{Ser}}$ without its long variable arm is an interesting question. Earlier studies suggested that mammalian $m$ SerRS can recognize not only $m t t^{2} A^{\text {Ser }}$ but also bacterial tRNA ${ }^{\text {Ser}}$; however, bacterial SerRS could not recognize mt tRNA $^{\text {Ser }}$ (Kumazawa etal., 1991). Also, mammalian mt SerRS recognizes the T-loop of both D-arm-lacking tRNA ${ }^{\text {Ser }}$ and cloverleaf-type $\mathrm{tRNA}^{\mathrm{Ser}}$ (UGA) without the long variable arm, and further requires a T-loop/D-loop interaction with tRNA $^{\text {Ser }}$ (UGA) (dual-mode recognition; Ueda et al., 1992; Shimada etal., 2001). The crystal structure of a mammalian mt SerRS, a model of it complexed with tRNA, and mutational analyses suggest that the N-terminal coiled-coil region, the distal helix, and the C-tail interact with the T-arm of mt tRNA ${ }^{\text {Ser }}$ (Chimnaronk etal., 2005, Figure 2). Mutational analysis of mammalian mt SerRS showed the substitution of some of the residues in $\mathrm{N}$-terminal coiled-coil region (shown in stick model in Figure 2B) reduced the aminoacylation activities of either one of two mt tRNAs ${ }^{\mathrm{Ser}}$ or both, suggesting that interaction of these residues with the tRNA in the enzyme-tRNA complex (Chimnaronk etal., 2005, Figure 2B). To maintain these interactions, the movement of $\mathrm{N}$-terminal coiled-coil region (shown as a red arrow) is expected (Chimnaronk et al., 2005, Figure 2B). Furthermore, mutational analysis of the enzyme suggested that, for the recognition of $\mathrm{tRNA}^{\mathrm{Ser}}$ (UGA) which have T-loop/D-loop interaction, Arg24, Tyr28 and Arg32, in the distal helix and the Lys93 and Arg122 on the N-terminal coiled-coil region are important (Figure $2 \mathbf{B}$ ). On the other hand, for the D-arm-lacking tRNA ${ }^{\text {Ser }}$ (GCU), Arg24 and Arg32 flanking the distal helix, and Arg129 on the N-terminal helical region are crucial (Figure 2B). Thus, with the dual-mode recognition, mammalian mt SerRS recognizes two tRNA ${ }^{\text {Ser }}$ isoacceptors which have different secondary structures using distinct sets of the residues.

Interestingly, in chromadorean nematode $\mathrm{mt}$, two tRNAs ${ }^{\mathrm{Ser}}$ have lost their D-arms, but the remaining 20 tRNAs have lost their T-arms. It is of interest whether nematode mt SerRS also recognize the $\mathrm{T}$-arm. If so, that may explain why only tRNAs ${ }^{\text {Ser }}$ have kept their T-arms in nematode mitochondria. However, it seems reasonable that the secondary structure of tRNA is governed by EF-Tu and ribosomes rather than aminoacyltRNA synthetase (ARS) during evolution, because the recognition mode of each ARS is constrained by only one or a few tRNA, while that of EF-Tu or the ribosome is constrained by over 20 tRNAs.

\section{EF-Tu}

The EF-Tu/GTP complex delivers aminoacyl-tRNAs to the A-site in ribosomes. Bacterial EF-Tu binds to the aminoacyl-moiety, a part of acceptor stem, and the T-arm (Nissen etal., 1995; Figure 3A), and it cannot bind to a tRNA analog missing the T-arm (Rudinger etal., 1994). In mitochondria, nuclearencoded EF-Tu exists. Due to the presence of aminoacyl-tRNAs missing the T-arm in nematode mitochondria (Watanabe et al., 1994; Ohtsuki et al., 1996), the EF-Tu counterpart in nematode mitochondria should use an alternative binding mode for $\mathrm{T}$ arm-lacking tRNAs. In fact, nematode mitochondria have two EF-Tu homologs, and only one of them (EF-Tu1) binds to T-arm-lacking tRNAs (Ohtsuki et al., 2001; Arita etal., 2006). 


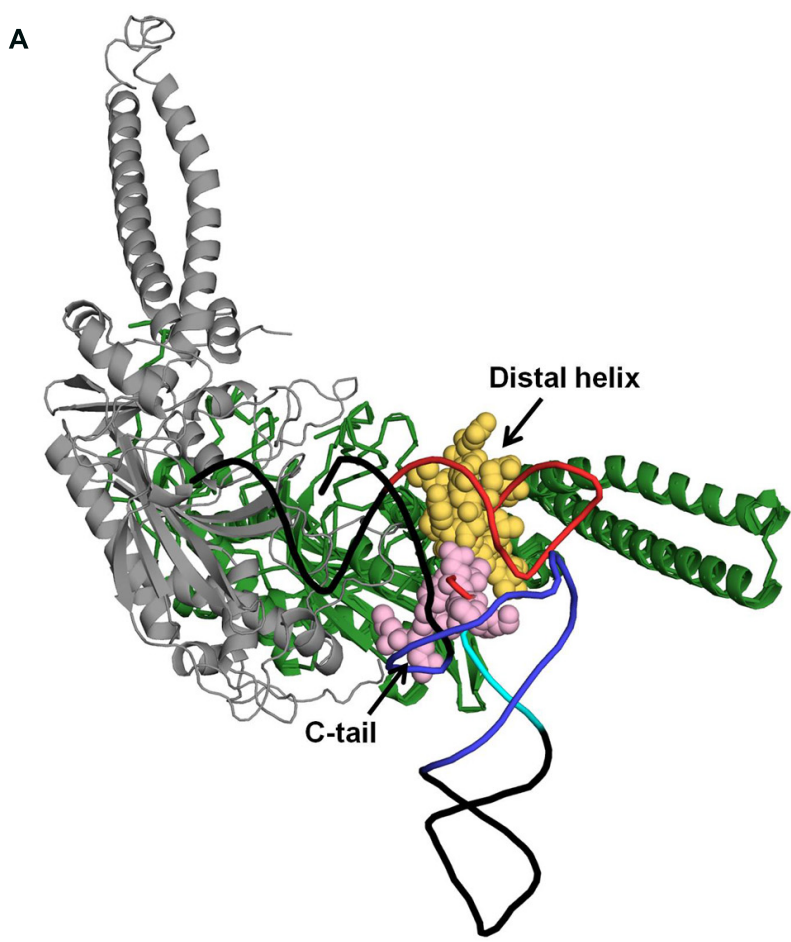

FIGURE 2 | (A) Docking model of mammalian mt SerRS and yeast tRNA ${ }^{\text {Phe }}$ (Chimnaronk etal., 2005). Two subunits of the enzyme are shown in green (monomer 1), and gray (monomer 2), respectively. Distal helix of monomer 1 and C-tail of monomer 2 which are mitochondrial-specific extensions and possibly interact with the tRNA, are shown in yellow and pink, respectively. In the tRNA, D-arm, variable loop, and T-arm are shown in purple, sky blue,

\section{B}

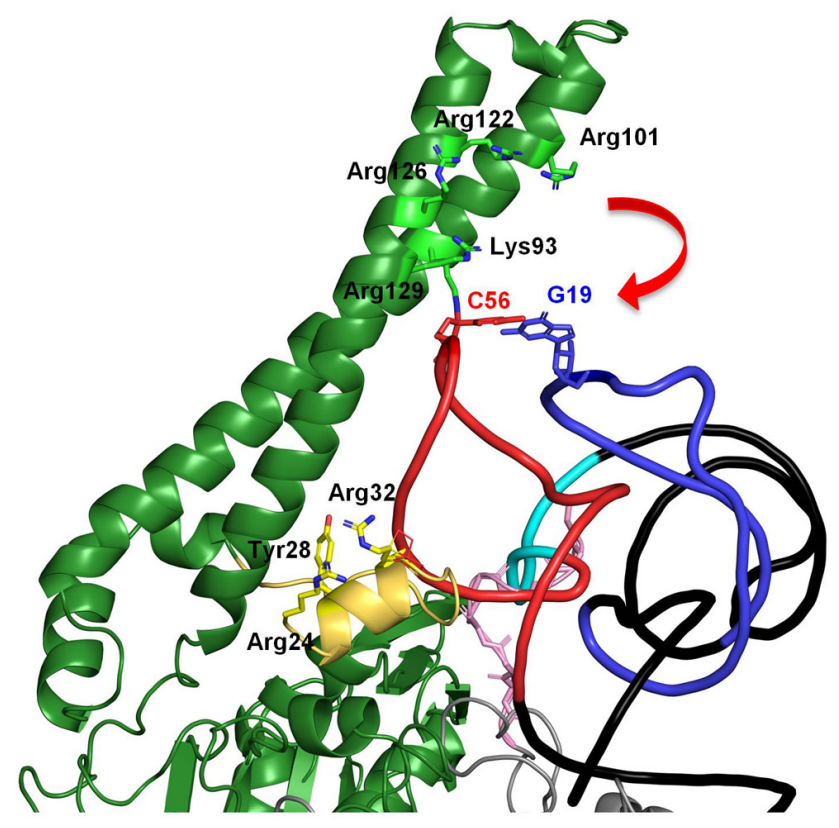

and red, respectively. (B) Putative interactions between the $\mathrm{N}$-terminal coiled-coil region and the C-tail distal helix with the T-arm of the tRNA. The interactions are inferred by mutational analysis of the enzyme (Chimnaronk et al., 2005). Residues in N-terminal coiled-coil region and distal helix involved in the interaction with tRNA are shown in stick model (Chimnaronk et al., 2005).
Caenorhabditis elegans $\mathrm{mt}$ EF-Tu1 has an approximately 60 aminoacid extension at the C-terminus (domain $3^{\prime}$ ) that is essential for binding to T-arm-lacking tRNAs (Ohtsuki et al., 2001; Sakurai et al., 2006). The extension likely interacts with the D-arm region of T-arm-lacking tRNAs through positive charges in Lys residues (Sakurai et al., 2006; Figures 3B,C). Interestingly, C. elegans mt EF-Tul lacks binding ability to cloverleaf-type tRNAs (Ohtsuki etal., 2001), which are missing in C. elegans mitochondria (Okimoto et al., 1992). In another lineage of nematode, Trichinella, mitochondria have T-arm-lacking tRNAs, cloverleaftype tRNAs, and D-arm-lacking tRNAs ${ }^{\mathrm{Ser}}$ (Lavrov and Brown, 2001). Trichinella also have two EF-Tu homologs, and one of them (EF-Tu1) binds to both T-arm-lacking tRNAs and cloverleaf-type tRNAs (Arita et al., 2006). Interestingly, Trichinella mt EF-Tu1 has a 41-residue C-terminal extension shorter than that in $C$. elegans mitochondria (Arita etal., 2006). A mutant of C. elegans mt EF-Tul with a 13-residue deletion at the C-terminus (43-residue extension left) cannot bind to T-arm-lacking tRNAs (Sakurai et al., 2006). Although the detailed tRNA binding mode of Trichinella mt EF-Tul has not been elucidated, it could be similar but not identical to that of C. elegans mt EF-Tu1. Note that C. elegans EF-Tu1 binds to only T-arm-lacking tRNAs, while Trichinella EF-Tu1 binds to T-arm-lacking tRNA, D-arm-lacking tRNA, and cloverleaf tRNA (Ohtsuki etal., 2001; Arita etal., 2006).
Nematode mitochondria have another EF-Tu homolog, EF-Tu2. Nematode mt EF-Tu2 has a short (about 15-residue) C-terminal extension, and it binds to D-arm-lacking tRNA ${ }^{\mathrm{Ser}}$, but not to T-arm-lacking or cloverleaf-type tRNAs (Ohtsuki et al., 2001; Suematsu et al., 2005; Arita et al., 2006). C. elegans mt EF-Tu2 binds to a region of the T-arm exposed due to the missing interaction between the T-arm and D-arm (Figures 3D,E; Suematsu et al., 2005). Interestingly, D-arm-lacking tRNAs in this species have anticodons for serine, and EF-Tu2 binds only to Ser-tRNA and accepts neither Ala-tRNA nor Val-tRNA with the same backbone (Ohtsuki et al., 2002b; Arita et al., 2006). This is likely due to the evolution of the aminoacyl-moiety binding pocket in EF-Tu2 to specialize in binding with the seryl moiety because of a unique adaptation in Ser-tRNA (Sato et al., 2006).

More recently, in some taxa other than the nematodes, such as Arthropoda, there have been mitochondrial T-arm-lacking tRNA genes discovered (Masta, 2000). Interestingly, there are two $\mathrm{mt}$ EF-Tu genes in arthropods (Ohtsuki and Watanabe, 2007). The functional differences between the two EF-Tu homologs in these species should be elucidated, and this project is in progress in our laboratory.

\section{FUTURE PERSPECTIVES}

Besides ARS and EF-Tu, other factors such as tRNA terminal nucleotidyltransferases (CCA enzymes) and ribosomes could 


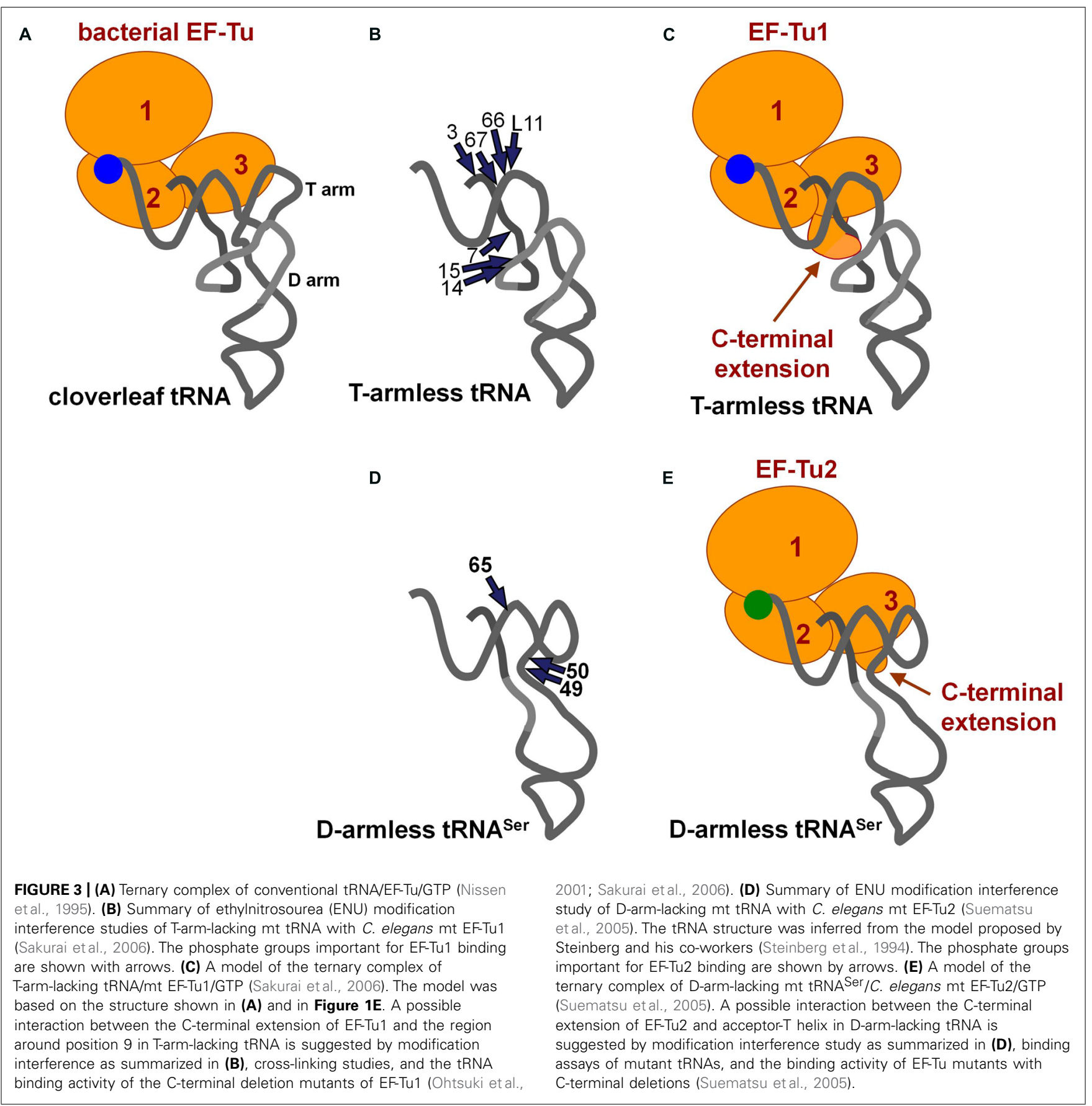

be interesting in terms of their interactions with shrunken $\mathrm{mt}$ tRNAs.

After the trimming of $3^{\prime}$ extra sequences, $3^{\prime} \mathrm{CCA}$ sequences are added to $3^{\prime}$ ends of pre-tRNAs by CCA enzymes (Deutscher, 1983). The bacterial CCA enzyme binds to the acceptor-T helix of pre-tRNA (Tomita etal., 2004), and thus a T-arm-lacking tRNA precursor is not a good substrate for the bacterial CCA enzyme (Tomari etal., 2002). On the other hand, the chromadorean nematode $C$. elegans has two genes for CCA enzymes, one of which encodes a putative mt CCA enzyme (Tomari et al., 2002). The recombinant (putative) mt CCA enzyme of C. elegans can recognize and efficiently add a CCA sequence, not only to conventional cloverleaf tRNAs, but also to T- or D-arm-lacking tRNAs (Tomari et al., 2002). It would be interesting to know how the nematode $\mathrm{mt}$ enzyme recognizes T-arm-lacking $\mathrm{mt}$ tRNAs efficiently.

During translation, conventional bacterial tRNA interacts with several sites in the ribosome (reviewed by Khade and Joseph, 2010). In bacterial ribosomes, the T-arm of P-site tRNA interacts with ribosomal protein L5 (Korostelev et al., 2006; Selmer et al., 2006). At the A-site, the T-arm of tRNA interacts with ribosomal protein L16 (Selmer et al., 2006; Voorhees et al., 2009). 
The residues in the A-site finger (helix 69) of 23S rRNA interact with the D-arm of tRNA at the A- and P-sites (Korostelev et al., 2006; Selmer et al., 2006; Voorhees et al., 2009). In a structural model of $C$. elegans $\mathrm{mt}$ rRNAs, the corresponding rRNA positions exist (Mears et al., 2002). At the E-site, residues in the $\mathrm{T}$ - and D-loops interact with ribosomal protein L1 and helices 76, 77, and 78 in $23 \mathrm{~S}$ rRNA (e.g., the L1 stalk; Korostelev et al., 2006; Selmer et al., 2006). Interestingly, the corresponding regions in nematode mt rRNA are missing (Mears et al., 2002). In general, mitochondrial ribosomal proteins are enlarged compared to their counterparts in bacteria (Koc et al., 2000; Suzuki et al., 2001), suggesting that mitochondrial ribosomal proteins may have alternate binding modes for truncated tRNAs. Further structural analysis of metazoan mt ribosomes (Sharma et al., 2003; Greber etal., 2014) would be helpful to reveal the detailed interaction mode between mt ribosomes and shrunken mt tRNAs.

Structural alterations of metazoan mt tRNAs have been compensated for by several interacting factors. The mode of compensation by these factors may explain why metazoan tRNAs have undergone truncation during evolution. Further investigation into the detailed binding modes between shrunken tRNAs and the interacting factors that co-evolved with them will shed light on how truncated tRNAs evolved.

\section{ACKNOWLEDGMENTS}

Our studies described in this review were supported by Ministry of Education, Culture, Sports, Science and Technology (MEXT), The Japan Society for the Promotion of Science (JSPS), the Kurata Memorial Hitachi Science and Technology Foundation and a grant from the Program for Promotion of Basic and Applied Researches for Innovations in Bio-oriented Industry (BRAIN), the Science and Technology Research Promotion Program for Agriculture, Forestry, Fisheries and Food Industry. We thank Prof. K. Watanabe and Prof. K. Kita for their continued support, and Dr. Sarin Chimnaronk for the docking model coordinates of mammalian mt SerRS/ tRNA.

\section{REFERENCES}

Arcari, P., and Brownlee, G. G. (1980). The nucleotide sequence of a small (3S) seryl-tRNA (anticodon GCU) from beef heart mitochondria. Nucleic Acids Res. 8, 5207-5212. doi: 10.1093/nar/8.22.5207

Arita, M., Suematsu, T., Osanai, A., Inaba, T., Kamiya, H., Kita, K., et al. (2006). An evolutionary 'intermediate state' of mitochondrial translation systems found in Trichinella species of parasitic nematodes: co-evolution of tRNA and EF-Tu. Nucleic Acids Res. 34, 5291-5929. doi: 10.1093/nar/ gkl526

Asahara, H., Himeno, H., Tamura, K., Nameki, N., Hasegawa, T., and Shimizu, M. (1994). Escherichia coli seryl-tRNA synthetase recognizes RNA $^{\text {Ser }}$ by its characteristic tertiary structure. J. Mol. Biol. 236, 738-748. doi: 10.1006/jmbi. 1994.1186

Baer, R. J., and Dubin, D. T. (1980). The sequence of a possible 5S RNA-equivalent in hamster mitochondria. Nucleic Acids Res. 8, 603-610. doi: 10.1093/nar/8. 16.3603

Biou, V., Yaremchuk, A., Tukalo, M., and Cusack, S. (1994). The $2.9 \AA$ crystal structure of $T$. thermophilus seryl-tRNA synthetase complexed with $\mathrm{tRNA}^{\mathrm{Ser}}$. Science 263, 1404-1410. doi: 10.1126/science.81 28220

Chihade, J. W., Hayashibara, K., Shiba, K., and Schimmel, P. (1998). Strong selective pressure to use G:U to mark an RNA acceptor stem for alanine. Biochemistry 37, 9193-9202. doi: 10.1021/bi9804636
Chimnaronk, S., Jeppesen, M. G., Suzuki, T., Nyborg, J., and Watanabe, K. (2005). Dual-mode recognition of noncanonical tRNAs ${ }^{\text {Ser }}$ by seryl-tRNA synthetase in mammalian mitochondria. EMBO J. 24, 3369-3379. doi: 10.1038/sj.emboj.7600811

de Bruijn, M. H., and Klug, A. (1983). A model for the tertiary structure of mammalian mitochondrial transfer RNAs lacking the entire 'dihydrouridine' loop and stem. EMBO J. 2, 1309-1321.

de Bruijn, M. H., Schreier, P. H., Eperon, I. C., Barrell, B. G., Chen, E. Y., Armstrong, P. W., et al. (1980). A mammalian mitochondrial serine transfer RNA lacking the "dihydrouridine" loop and stem. Nucleic Acids Res. 8, 5213-5222. doi: 10.1093/nar/8.22.5213

Deutscher, M. P. (1983). "tRNA nucleotidyltransferase and the-C-C-A terminus of transfer RNA," in Enzymes of Nucleic Acid Synthesis and Modification, Vol. 2, RNA Enzymes, ed. S.T. Jacob (Boca Raton, FL: CRC Press), 159-183.

Dubin, D. T., and Friend, D. A. (1972). Comparison of cytoplasmic and mitochondrial 4 S RNA from cultured hamster cells: physical and metabolic properties. J. Mol. Biol. 71, 163-175. doi: 10.1016/0022-2836(72)90344-0

Dubin, D. T., Jones, T. N., and Cleaves, G. R. (1974). An unmethylated “3 SE” RNA in hamster mitochondria: a 5 S RNA-equivalent? Biochem. Biophys. Res. Commun. 56, 401-406. doi: 10.1016/0006-291X(74)90856-0

Frazer-Abel, A. A., and Hagerman, P. J. (1999). Determination of the angle between the acceptor and anticodon stems of a truncated mitochondrial tRNA. J. Mol. Biol. 285, 581-593. doi: 10.1006/jmbi.1998.2320

Frazer-Abel, A. A., and Hagerman, P. J. (2004). Variation of the acceptor-anticodon interstem angles among mitochondrial and non-mitochondrial tRNAs. J. Mol. Biol. 343, 313-325. doi: 10.1016/j.jmb.2004.07.087

Frazer-Abel, A. A., and Hagerman, P. J. (2008). Core flexibility of a truncated metazoan mitochondrial tRNA. Nucleic Acids Res. 36, 5472-5481. doi: 10.1093/nar/gkn529

Giegé, R., Sissler, M., and Florentz, C. (1998). Universal rules and idiosyncratic features in tRNA identity. Nucleic Acids Res. 26, 5017-5035. doi: $10.1093 /$ nar/26.22.5017

Greber, B. J., Boehringer, D., Leitner, A., Bieri, P., Voigts-Hoffmann, F., Erzberger, J. P., et al. (2014) Architecture of the large subunit of the mammalian mitochondrial ribosome. Nature 505, 515-519. doi: 10.1038/nature 12890

Hanada, T., Suzuki, T., Yokogawa, T., Takemoto-Hori, C., Sprinzl, M., and Watanabe, K. (2001). Translation ability of mitochondrial tRNAs ${ }^{\text {Ser }}$ with unusual secondary structures in an in vitro translation system of bovine mitochondria. Genes Cells 6, 1019-1030. doi: 10.1046/j.1365-2443.2001. 00491.x

Hayashi, I., Yokogawa, T., Kawai, G., Ueda, T., Nishikawa, K., and Watanabe, K. (1997). Assignment of imino proton signals of G-C base pairs and magnesium ion binding: an NMR study of bovine mitochondrial tRNA ${ }^{\mathrm{Ser}}$ (GCU) lacking the entire D arm. J. Biochem. 121, 1115-1122. doi: 10.1093/oxfordjournals.jbchem.a021703

Jühling, F., Mörl, M., Hartmann, R. K., Sprinzl, M., Stadler, P. F., and Pütz, J. (2009). tRNAdb 2009: compilation of tRNA sequences and tRNA genes. Nucleic Acids Res. 37, D159-D162. doi: 10.1093/nar/gkn772

Jühling, F., Pütz, J., Bernt, M., Donath, A., Middendorf, M., Florentz, C., et al. (2012a). Improved systematic tRNA gene annotation allows new insights into the evolution of mitochondrial tRNA structures and into the mechanisms of mitochondrial genome rearrangements. Nucleic Acids Res. 40, 2833-2845. doi: 10.1093/nar/gkr1131

Jühling, F., Pütz, J., Florentz, C., and Stadler, P. F. (2012b). Armless mitochondrial tRNAs in Enoplea (Nematoda). RNA Biol. 9, 1161-1166. doi: 10.4161/rna. 21630

Khade, P., and Joseph, S. (2010). Functional interactions by transfer RNAs in the ribosome. FEBS Lett. 584, 420-426. doi: 10.1016/j.febslet.2009. 11.034

Koc, E. C., Burkhart, W., Blackburn, K., Moseley, A., Koc, H., and Spremulli, L. L. (2000). A proteomics approach to the identification of mammalian mitochondrial small subunit ribosomal proteins. J. Biol. Chem. 275, 32585-33291. doi: 10.1074/jbc.M003596200

Korostelev, A., Trakhanov, S., Laurberg, M., and Noller, H. F. (2006). Crystal structure of a $70 \mathrm{~S}$ ribosome-tRNA complex reveals functional interactions and rearrangements. Cell 126, 1065-1077. doi: 10.1016/j.cell.2006. 08.032 
Kumazawa, Y., Himeno, H., Miura, K., and Watanabe, K. (1991). Unilateral aminoacylation specificity between bovine mitochondria and eubacteria. J. Biochem. 109, 421-427.

Lavrov, D. V., and Brown, W. M. (2001). Trichinella spiralis mtDNA: a nematode mitochondrial genome that encodes a putative ATP8 and normally structured tRNAs and has a gene arrangement relatable to those of coelomate metazoans. Genetics 157, 621-637.

Lovato, M. A., Chihade, J. W., and Schimmel, P. (2001). Translocation within the acceptor helix of a major tRNA identity determinant. EMBO J. 20, 4846-4853. doi: 10.1093/emboj/20.17.4846

Masta, S. E. (2000). Mitochondrial sequence evolution in spiders: intraspecific

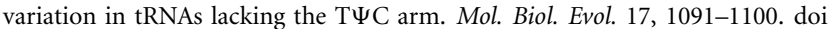
10.1093/oxfordjournals.molbev.a026390

Mears, J. A., Cannone, J. J., Stagg, S. M., Gutell, R. R., Agrawal, R. K., and Harvey, S. C. (2002). Modeling a minimal ribosome based on comparative sequence analysis. J. Mol. Biol. 321, 215-234. doi: 10.1016/S0022-2836(02) 00568-5

Nissen, P., Kjeldgaard, M., Thirup, S., Polekhina, G., Reshetnikova, L., Clark, B. F., et al. (1995). Crystal structure of the ternary complex of Phe-tRNA ${ }^{\text {Phe }}$ EF-Tu, and a GTP analog. Science 270, 1464-1472. doi: 10.1126/science.270.52 41.1464

Nureki, O., Niimi, T., Muramatsu, T., Kanno, H., Kohno, T., Florentz, C., etal. (1994). Molecular recognition of the identity-determinant set of isoleucine transfer RNA from Escherichia coli. J. Mol. Biol. 236, 710-724. doi: 10.1006/jmbi.1994.1184

Ohtsuki, T., Kawai, G., and Watanabe, K. (1998). Stable isotope-edited NMR analysis of Ascaris suum mitochondrial tRNA ${ }^{\text {Met }}$ having a TVreplacement loop. J. Biochem. 124, 28-34. doi: 10.1093/oxfordjournals.jbchem. a022092

Ohtsuki, T., Kawai, G., Watanabe, Y., Kita, K., Nishikawa, K., and Watanabe, K. (1996). Preparation of biologically active Ascaris suum mitochondrial tRNA ${ }^{\text {Met }}$ with a TV-replacement loop by ligation of chemically synthesized RNA fragments. Nucleic Acids Res. 24, 662-667. doi: 10.1093/nar/24. 4.662

Ohtsuki, T., Kawai, G., and Watanabe, K. (2002a). The minimal tRNA: unique structure of Ascaris suum mitochondrial tRNA ${ }^{\text {Ser }}$ (UCU) having a short T arm and lacking the entire D arm. FEBS Lett. 514, 37-43. doi: 10.1016/S00145793(02)02328-1

Ohtsuki, T., Sato, A., Watanabe, Y., and Watanabe, K. (2002b). A unique serinespecific elongation factor Tu found in nematode mitochondria. Nat. Struct. Biol. 9, 669-673. doi: 10.1038/nsb826

Ohtsuki, T., and Watanabe, Y. (2007). T-armless tRNAs and elongated elongation factor Tu. IUBMB Life 59, 68-75. doi: 10.1080/15216540701 218722

Ohtsuki, T., Watanabe, Y., Takemoto, C., Kawai, G., Ueda, T., Kita, K., et al. (2001) An "elongated" translation elongation factor Tu for truncated tRNAs in nematode mitochondria. J. Biol. Chem. 276, 21571-21577. doi: 10.1074/jbc.M0111 18200

Okimoto, R., Macfarlane, J. L., Clary, D. O., and Wolstenholme, D. R. (1992). The mitochondrial genomes of two nematodes, Caenorhabditis elegans and Ascaris suum. Genetics 130, 471-498.

Rubio, M. A., Rinehart, J. J., Krett, B., Duvezin-Caubet, S., Reichert, A. S., Söll, D., et al. (2008). Mammalian mitochondria have the innate ability to import tRNAs by a mechanism distinct from protein import. Proc. Natl. Acad. Sci. U.S.A. 105, 9186-9191. doi: 10.1073/pnas.0804283105

Rudinger, J., Blechschmidt, B., Ribeiro, S., and Sprinzl, M. (1994). Minimalist aminoacylated RNAs as efficient substrates for elongation factor Tu. Biochemistry 33, 5682-5688. doi: 10.1021/bi00185a003

Sakurai, M., Ohtsuki, T., Suzuki, T., and Watanabe, K. (2005a). Unusual usage of wobble modifications in mitochondrial tRNAs of the nematode Ascaris suum. FEBS Lett. 579, 2767-2772. doi: 10.1016/j.febslet.2005. 04.009

Sakurai, M., Ohtsuki, T., and Watanabe, K. (2005b). Modification at position 9 with 1-methyladenosine is crucial for structure and function of nematode mitochondrial tRNAs lacking the entire T-arm. Nucleic Acids Res. 33, 1653-1661. doi 10.1093/nar/gki309

Sakurai, M., Watanabe, Y., Watanabe, K., and Ohtsuki, T. (2006). A protein extension to shorten RNA: elongated elongation factor-Tu recognizes the D-arm of
T-armless tRNAs in nematode mitochondria. Biochem. J. 399, 249-256. doi: 10.1042/BJ20060781

Sato, A., Watanabe, Y., Suzuki, T., Komiyama, M., Watanabe, K., and Ohtsuki, T. (2006). Identification of the residues involved in the unique serine specificity of Caenorhabditis elegans mitochondrial EF-Tu2. Biochemistry 45, 10920-10927. doi: 10.1021/bi060536i

Selmer, M., Dunham, C. M., Murphy, F. V. IV, Weixlbaumer, A., Petry, S., Kelley, A. C., et al. (2006). Structure of the 70 S ribosome complexed with mRNA and tRNA. Science 313, 1935-1942. doi: 10.1126/science.1131127

Sharma, M. R., Koc, E. C., Datta, P. P., Booth, T. M., Spremulli, L. L., and Agrawal, R. K. (2003). Structure of the mammalian mitochondrial ribosome reveals an expanded functional role for its component proteins. Cell 115, 97-108. doi: 10.1016/S0092-8674(03)00762-1

Shimada, N., Suzuki, T., and Watanabe, K. (2001). Dual mode recognition of two isoacceptor tRNAs by mammalian mitochondrial seryl-tRNA synthetase. J. Biol. Chem. 276, 46770-46778. doi: 10.1074/jbc.M105150200

Seutin, G., Lang, B. F., Mindell, D. P., and Morais, R. (1994). Evolution of the WANCY region in amniote mitochondrial DNA. Mol. Biol. Evol. 11, 329-340.

Steinberg, S., Gautheret, D., and Cedergren, R. (1994). Fitting the structurally diverse animal mitochondrial tRNAs ${ }^{\mathrm{Ser}}$ to common three-dimensional constraints. J. Mol. Biol. 236, 982-989. doi: 10.1016/0022-2836(94)90004-3

Suematsu, T., Sato, A., Sakurai, M., Watanabe, K., and Ohtsuki, T. (2005). A unique tRNA recognition mechanism of Caenorhabditis elegans mitochondrial EF-Tu2. Nucleic Acids Res. 33, 4683-4691. doi: 10.1093/nar/ gki784

Suzuki, T., Terasaki, M., Takemoto-Hori, C., Hanada, T., Ueda, T., Wada, A., et al. (2001). Proteomic analysis of the mammalian mitochondrial ribosome. Identification of protein components in the $28 \mathrm{~S}$ small subunit. J. Biol. Chem. 276, 33181-33195. doi: 10.1074/jbc.M103236200

Tomari, Y., Suzuki, T., and Ueda, T. (2002). tRNA recognition by CCAadding enzyme. Nucleic Acids Res. Suppl. 2002, 77-78. doi: 10.1093/nass/ 2.1.77

Tomita, K., Fukai, S., Ishitani, R., Ueda, T., Takeuchi, N., Vassylyev, D. G., et al. (2004). Structural basis for template-independent RNA polymerization. Nature 430, 700-704. doi: 10.1038/nature02712

Ueda, T., Ohta, T., and Watanabe, K. (1985). Large scale isolation and some properties of AGY-specific serine tRNA from bovine heart mitochondria. J. Biochem $98,1275-1284$.

Ueda, T., Yotsumoto, Y., Ikeda, K., and Watanabe, K. (1992). The T-loop region of animal mitochondrial tRNA ${ }^{\mathrm{Ser}}(\mathrm{AGY})$ is a main recognition site for homologous seryl-tRNA synthetase. Nucleic Acids Res. 20, 2217-2222. doi: 10.1093/nar/20.9.2217

Voorhees, R. M., Weixlbaumer, A., Loakes, D., Kelley, A. C., and Ramakrishnan, V. (2009). Insights into substrate stabilization from snapshots of the peptidyl transferase center of the intact 70S ribosome. Nat. Struct. Mol. Biol. 16, 528-533. doi: 10.1038/nsmb.1577

Watanabe, Y., Tsurui, H., Ueda, T., Furushima, R., Takamiya, S., Kita, K., et al. (1994). Primary and higher order structures of nematode (Ascaris suum) mitochondrial tRNAs lacking either the T or D stem. J. Biol. Chem. 269, 2290222906.

Watanabe, Y., Tsurui, H., Ueda, T., Furushima-Shimogawara, R., Takamiya, S., Kita, K., et al. (1997). Primary sequence of mitochondrial tRNA ${ }^{\text {Arg }}$ of a nematode Ascaris suum: occurrence of unmodified adenosine at the first position of the anticodon. Biochim. Biophys. Acta 1350, 119-122. doi: 10.1016/S01674781(96)00211-4

Wende, S., Platzer, E. G., Jühling, F., Pütz, J., Florentz, C., Stadler, P. F., et al. (2014). Biological evidence for the world's smallest tRNAs. Biochimie 100, 151-158. doi: 10.1016/j.biochi.2013.07.034

Wolstenholme, D. R., Macfarlane, J. L., Okimoto, R., Clary, D. O., and Wahleithner, J. A. (1987). Bizarre tRNAs inferred from DNA sequences of mitochondrial genomes of nematode worms. Proc. Natl. Acad. Sci. U.S.A. 84, 1324-1328. doi: 10.1073/pnas.84.5.1324

Wolstenholme, D. R., Okimoto, R., and Macfarlane, J. L. (1994). Nucleotide correlations that suggest tertiary interactions in the TV-replacement loop-containing mitochondrial tRNAs of the nematodes, Caenorhabditis elegans and Ascaris suum. Nucleic Acids Res. 22, 4300-4306. doi: 10.1093/nar/22.20.4300

Yokogawa, T., Kumazawa, Y., Miura, K., and Watanabe, K. (1989). Purification and characterization of two serine isoacceptor tRNAs from bovine mitochondria 
by using a hybridization assay method. Nucleic Acids Res. 17, 2623-2638. doi: 10.1093/nar/17.7.2623

Yokogawa, T., Shimada, N., Takeuchi, N., Benkowski, L., Suzuki, T., Omori, A., etal. (2000). Characterization and tRNA recognition of mammalian mitochondrial seryl-tRNA synthetase. J. Biol. Chem. 275, 19913-19920. doi: 10.1074/jbc.M908473199

Conflict of Interest Statement: The authors declare that the research was conducted in the absence of any commercial or financial relationships that could be construed as a potential conflict of interest.

Received: 31 January 2014; accepted: 12 April 2014; published online: 01 May 2014.
Citation: Watanabe Y-i, Suematsu T and Ohtsuki T (2014) Losing the stem-loop structure from metazoan mitochondrial tRNAs and co-evolution of interacting factors. Front Genet. 5:109. doi: 10.3389/fgene.2014.00109

This article was submitted to Non-Coding RNA, a section of the journal Frontiers in Genetics.

Copyright (c) 2014 Watanabe, Suematsu and Ohtsuki. This is an open-access article distributed under the terms of the Creative Commons Attribution License (CC BY). The use, distribution or reproduction in other forums is permitted, provided the original author(s) or licensor are credited and that the original publication in this journal is cited, in accordance with accepted academic practice. No use, distribution or reproduction is permitted which does not comply with these terms 